\title{
Le dromadaire et le cochon : deux visions opposées de l'élevage?
}

\author{
Bernard Faye, Vincent Porphyre \\ Zootechniciens, CIRAD, UR18 Systèmes d'élevage en milieux méditerranéens et tropicaux, 34398 Montpellier cedex, France
}

\begin{abstract}
Les rapports entre l'homme et l'animal font l'objet de nombreux travaux récents dont la revue rend régulièrement compte. Que ce soit en anthropologie, en philosophie ou en zootechnie, les recherches et débats passionnés, voire militants, que cette thématique suscite concernent les sociétés des pays du Nord. Dans ce contexte, l'intérêt de l'article tient d'abord à son champ géographique centré sur les pays du Sud. Rien d'uniforme pourtant dans les rapports des sociétés du Sud avec des animaux domestiques, comme le démontrent les exemples extrêmes du dromadaire et du cochon. Mais, en même temps, rien d'irrémédiablement opposé non plus. Au-delà des approches spécialisées de la zootechnie, les auteurs proposent un tableau déjà pluridisciplinaire des deux types d'élevage, par des ouvertures à des enjeux sociologiques et économiques, mais également écologiques.
\end{abstract}

La Rédaction

\section{Mots-clés :}

porcin ;

camélidé ;

élevage ;

développement ;

zootechnie

\section{Keywords:}

pigs;

camels;

livestock farming;

development;

zootechnics
Résumé - Le dromadaire et le cochon sont deux espèces d'élevage emblématiques d'un gradient allant de l'extensif à l'intensif et se déclinant sur divers registres a priori exclusifs. Pourtant, malgré les différences fondamentales de ces deux espèces, tant sur les plans physiologique et zootechnique que sociologique et culturel, leur élevage et les filières dont ils sont à l'origine sont confrontés à des enjeux parfois comparables (qualité des produits, durabilité économique, biodiversité, impact environnemental, marché régional) et à des dynamiques (intensification, diversification) qui tendraient à les rapprocher en termes de développement et sur lesquelles un effort de recherche s'avère nécessaire. Le présent article est né de la confrontation de deux zootechniciens, chacun spécialiste d'une espèce donnée.

\section{Introduction}

L'activité d'élevage concerne, à travers le monde, un nombre assez peu important d'espèces animales comparé au nombre total d'espèces inventoriées (Digard, 2009). Parmi elles, tout semble a priori opposer le dromadaire et le cochon. L'hypothèse selon laquelle ces deux espèces sont aux antipodes des visions du développement de l'élevage s'appuie sur les présupposés des oppositions sur les plans tant biologique que zootechnique, géographique et social.

Les connotations idéologiques liées à la situation politique internationale ont pu brouiller parfois les images $\mathrm{du}$ dromadaire et $\mathrm{du}$ cochon, le chameau «islamique »

Auteur correspondant : B. Faye, faye@cirad.fr 
opposé au porc «chrétien », reproduisant le mythe du « conflit des civilisations » (Huntington, 1997). C'est ainsi qu'on a pu accuser les chercheurs irakiens, avant la deuxième guerre contre l'Irak, de préparer des armes biologiques à partir du virus de la variole du chameau (camelpox) dont le génome s'est révélé très proche de celui de la variole humaine. Un article du New Scientist affirmait que cette proximité génomique laissait suggérer «que le virus du chameau possède déjà les gènes nécessaires pour devenir pathogène pour l'homme » (Coghlan et Mackenzie, 2002). Un autre exemple est celui des dromadaires qui, s'approchant trop près des campements militaires à Djibouti, sont victimes des tirs de sommation, comme s'en est émue la presse locale (Faye, 2003b). Le cochon reste, lui, source de discorde et d'échanges virulents entre chrétiens, juifs et musulmans. En 2007, année du cochon dans le calendrier lunaire, la Chine, pour ménager les minorités et les pays musulmans voisins, a demandé aux responsables du secteur industriel d'éviter d'utiliser l'image de cet animal lors des célébrations, les obligeant à revoir leurs campagnes de communication publicitaire. L'image du cochon touche aussi les thèmes politiques: Orwell (1945), dans La Ferme des animaux, dépeint les révolutionnaires sous les traits de cochons gras et corrompus; c'est avec un visage de cochon que Spiegelman (1991) dessine ses personnages polonais, leur reprochant un antisémitisme plus ou moins explicite.

Toutefois, ces connotations symboliques n'épuisent pas les questions sous-jacentes qui se posent aux éleveurs des pays en développement. Les élevages du dromadaire et du cochon sont certes soumis à des contraintes qui leur sont spécifiques, les dynamiques de leur évolution sont probablement peu comparables et les enjeux de durabilité qui les sous-tendent ne sont pas de même nature. Pour autant, les questions de recherche et de développement qui les concernent sont-elles aussi dissemblables, puisqu'on oppose facilement, en évoquant ces deux espèces, système extensif et système intensif, mode de vie nomade et sédentarité ? Le présent article est le fruit d'une réflexion croisée entre deux zootechniciens - l'un, « camélologue »; l'autre, « suinologue »-confrontés au développement de ces filières dans les pays du Sud.

\section{Situation et idées reçues : des élevages radicalement différents ?}

\section{Les profondeurs historiques d'une rencontre improbable}

Le dromadaire, dans sa forme actuelle, a été probablement domestiqué il y a environ 4000 ans BP dans le sud de la péninsule d'Arabie. D'après Uerpman et Uerpman (2002), une forme sauvage de dromadaire était encore chassée dans l'est de la péninsule (actuels Émirats arabes unis) il y a environ 5000 ans BP, témoignant d'un long processus de domestication étalé sur un millénaire au moins. La première mention historique du dromadaire est d'ordre militaire, puisque sa présence est signalée 1100 ans av. J.-C. lors d'une bataille entreles tribus arabes du nord de la péninsule et les populations méditerranéennes. Aussi peut-on penser que les premières utilisations du dromadaire n'étaient pas tant pour la production de lait et de viande que pour sa capacité à être monté ou à porter. Depuis son aire d'origine, le dromadaire a occupé les régions de l'Ancien Monde en voie d'aridification, la Corne de l'Afrique d'abord (vers 1000 ans av. J.C.), puis l'Afrique saharienne et sahélienne vers l'ouest (au début de l'ère chrétienne), le MoyenOrient et jusqu'à l'Inde vers l'est à l'occasion des invasions d'Alexandre le Grand.

Visiblement très lié aux conquêtes, notamment celles de l'islam au moment de son expansion vers le nord de l'Afrique, le dromadaire se retrouve jusque dans le sud de l'Espagne et en Sicile tout au long du Moyen Âge. Pourtant, à côté de son usage guerrier des premiers temps, de nombreux témoignages archéologiques attestent de son utilisation laitière ancienne (Vigne, 2011). Il n'en reste pas moins que, dès sa domestication, le dromadaire avait trouvé une place parmi les hommes moins pour les nourrir que pour les transporter ou porter leurs biens. Il était donc voué dès les premiers temps à la mobilité.

L'histoire du cochon est radicalement différente. Sa domestication est plus ancienne que celle du dromadaire (VII ${ }^{\mathrm{e}}$ millénaire av. J.-C.) ; mais, l'animal étant incapable de suivre des groupes humains nomades, elle coïncide avec la sédentarisation des hommes et l'apparition de l'agriculture. Actuellement, les archéologues s'accordent pour penser qu'il y a eu plusieurs foyers de domestication, probablement en Asie Mineure (l'actuelle Turquie), puis plus tard en Chine (Vigne, 2011). De par sa facilité d'élevage et sa productivité, le cochon va vite progresser vers l'Europe et le reste de l'Asie, mais son inadaptation aux contextes arides le maintiendra hors des zones à élevage mobile.

\section{Places respectives dans le monde}

L'examen de la carte mondiale de répartition des deux espèces permet d'emblée de vérifier une première évidence: leurs distributions ne se recoupent que très partiellement(Fig. 1 et 2). Cette séparation spatiale est liée à deux facteurs principaux d'ordre climatique et culturel : le dromadaire, espèce hyperadaptée aux conditions arides, est l'animal des déserts de l'Ancien Monde, où est né l'islam, ce qui explique sa présence majoritairement dans les pays musulmans, dans lesquels le porc, animal associé à un tabou alimentaire religieux, est quasiment absent ; a contrario, le porc, animal du sédentaire par excellence, ne saurait s'adapter aux maigres ressources 


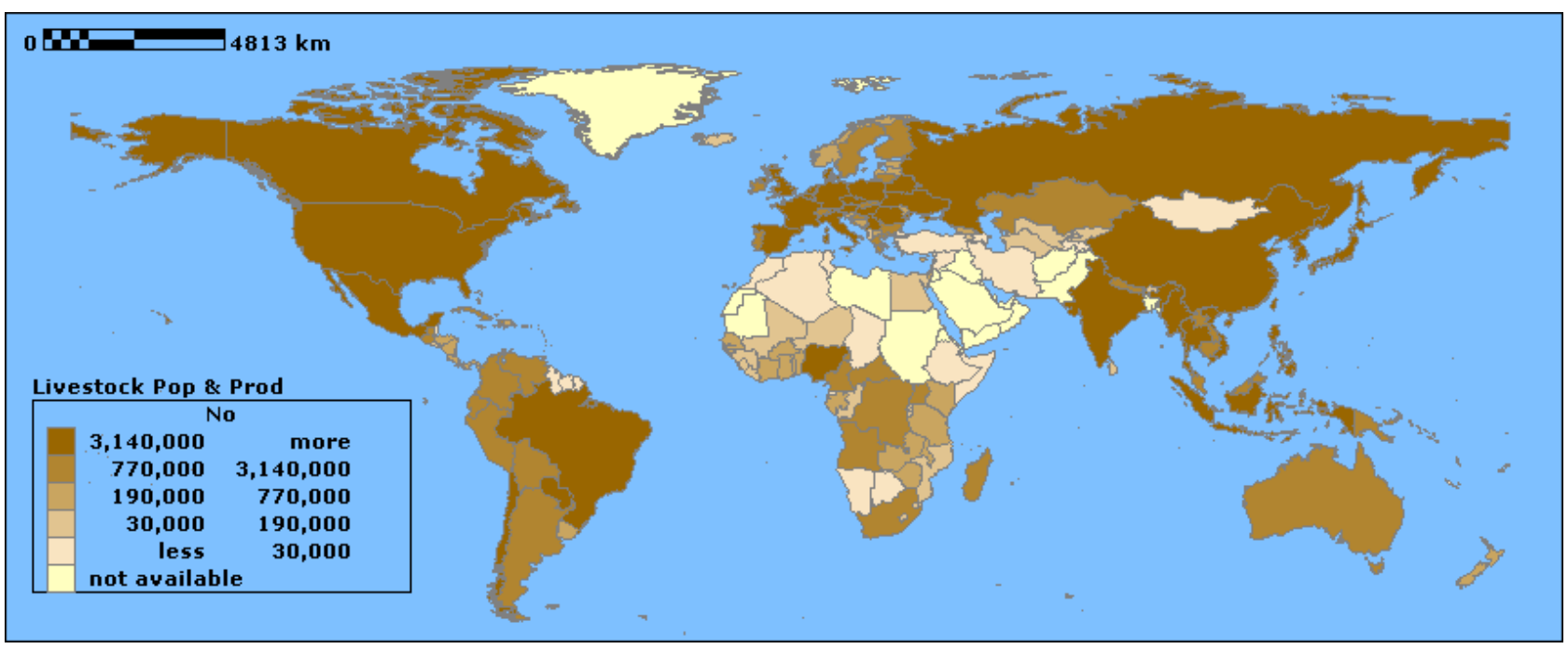

Fig. 1. Population porcine dans le monde en 2004 (source FAOSTAT).

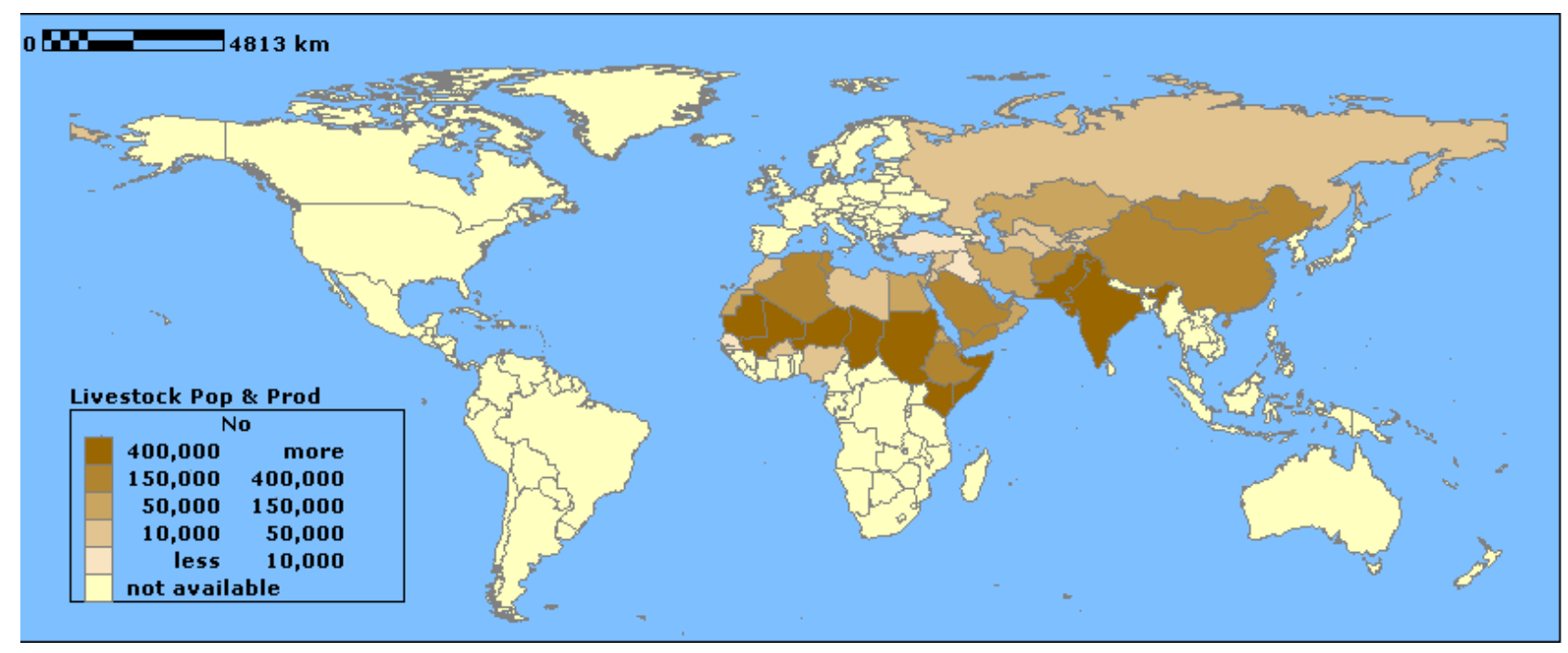

Fig. 2. Population cameline dans le monde en 2003 (source FAOSTAT).

des zones arides, et il est par ailleurs attaché à la consommation carnée des chrétiens, bouddhistes et animistes. Au-delà de l'opposition nomade-sédentaire, la répartition des deux espèces est donc en partie superposable à celle des deux grandes religions du Livre, islam et christianisme. Il conviendrait d'ajouter que le porc est aussi l'objet de tabou alimentaire dans le judaïsme, l'hindouisme et chez les chrétiens monophysites d'Éthiopie. Toutefois, son élevage est répandu partout où les communautés religieuses cohabitent. On le pratique ainsi en Chine, en Inde, en Asie centrale, en Afrique de l'Ouest côtière, en Indonésie ou en Malaisie, bien que les communautés musulmanes ou hindouistes y soient importantes. Il y occupe des espaces régionaux différents de ceux consacrés à l'élevage camelin au sein de mêmes entités nationales.
$\mathrm{Du}$ fait de ces contraintes religieuses, les zones de consommation et de transformation des produits issus de chaque espèce recouvrent totalement les zones de production. Le marché international des produits camelins est peu développé et ne concerne que des ensembles régionaux. Les flux commerciaux concernent essentiellement la viande, le lait de chamelle étant limité aux circuits locaux. Les flux commerciaux les plus importants pour l'espèce cameline se structurent des pays sahéliens vers le Maghreb et de la Corne de l'Afrique vers la péninsule d'Arabie (Alary et Faye, 2009).

En revanche, le commerce international de la viande de porc est bien développé. Les principaux flux internationaux concernent les pays occidentaux ainsi que les principales zones de consommation en Asie (Chine, Japon). Le porc est la viande la plus largement 
consommée dans le monde. La Chine en est le plus gros producteur (50\% du cheptel mondial, en constante augmentation) et le plus gros consommateur. L'Europe vient en seconde position, fournissant le plus grand nombre de porcs charcutiers. Puis vient la Russie, avec une production stable mais un marché en croissance. Les États-Unis tendent à augmenter leurs exportations, profitant de la faiblesse relative du dollar. Le plus gros importateur de viande de porc est le Japon. En Afrique, les flux restent régionaux ( $\mathrm{du}$ Tchad vers le Cameroun, par exemple), contraints par des situations zoosanitaires difficiles (Mopaté et al., 2006 ; Koussou et Duteurtre, 2003).

\section{Rapport à l'espace et utilisation des ressources alimentaires}

Le dromadaire, animal herbivore, ne survit qu'en valorisant par un pâturage ambulatoire des ressources herbacées ou ligneuses sur des territoires très vastes tant la densité de la biomasse y est faible (Longo-Hammouda et al., 2007). C'est donc un animal voué à la mobilité, en adéquation avec le mode de vie nomade des chameliers. Le porc, à l'inverse, est un omnivore, voire un détritivore efficace. Piètre marcheur, il ne s'épanouit que dans un milieu lui offrant suffisamment de nourritures riches en protéines dans un rayon restreint. Il ne se déplace généralement pas et, plus qu'aucune autre espèce, il se complait dans le « hors-sol ». Cependant, les porcs ensauvagés sont capables d'envahir les écosystèmes forestiers, insulaires ou de savanes (Brésil, Australie, NouvelleCalédonie) et d'y proliférer.

L'accès aux ressources construit un rapport différencié à l'espace, bien que les évolutions en cours laissent la possibilité de toute la gamme des modes d'élevage pour les deux espèces. Ainsi, si le modèle prégnant est l'élevage porcin hors-sol en bâtiment, il existe aussi des élevages en plein air, des systèmes basés sur la divagation et différents degrés d'élevage en claustration selon l'espace disponible et les apports alimentaires. Évidemment, une gamme aussi large est observable chez les dromadaires, mais avec une nette prépondérance du système extensif, où l'absence de gardiennage conduit souvent à une divagation des troupeaux.

Finalement, on peut relever au sein des deux espèces une opposition entre des systèmes concentrationnaires (unités industrielles de porcs, unités camelines laitières dans les pays du Golfe) et systèmes « atomisés » (élevages familiaux). Toutefois, malgré le manque de statistiques précises sur ces aspects, le type concentrationnaire nous semble être plutôt la marque de l'élevage porcin et le type dispersé, celle de l'élevage camelin.

Du fait de cette différence d'accès et bien que les élevages porcins soient beaucoup plus nombreux que les camelins, les surfaces qu'ils occupent à l'échelle de la planète sont presque du même ordre de grandeur. Une estimation grossière permet d'affirmer que les 25 millions de grands camélidés se répartissent sur 35 millions de kilomètres carrés, soit sur presque toutes les étendues arides de l'Ancien Monde (sauf l'Afrique australe), alors que les 920 millions de porcs occupent approximativement 50 millions de kilomètres carrés.

Différence de densité, donc, mais différence aussi dans la concurrence alimentaire avec l'homme. Si le dromadaire valorise surtout des espaces où l'agriculture est absente (hors oasis), le cochon partage l'intimité alimentaire de l'homme, soit en se nourrissant traditionnellement de ses déchets de cuisine ou des résidus de récolte, soit, dans un contexte "moderne ", en consommant des céréales pouvant être destinées à l'alimentation humaine. Toutefois, des recherches sur l'utilisation des ressources agroforestières riches en fibres font valoir la grande capacité des porcs à occuper un territoire en valorisant des végétations ligneuses, agrémentées de racines et de fruits (Leterme et al., 2007).

\section{Incomparables productivités}

On ne s'attardera pas ici sur les différences évidentes de physiologie entre un dromadaire, entièrement tourné vers l'économie et le recyclage des éléments vitaux (Yagil, 1985 ; Faye et Bengoumi, 2000 ; Bengoumi et Faye, 2002), une faible productivité numérique, une croissance lente, compensée par une remarquable longévité, et un cochon, espèce prolifique, à croissance rapide, convertisseur remarquable des résidus agroalimentaires, mais à l'inverse peu adaptée à la chaleur et encore moins à la déshydratation. De ces physiologies, découlent des productivités très éloignées les unes des autres. Un porc en croissance gagne de 300 à $800 \mathrm{~g} / \mathrm{j}$ en phase d'engraissement, soit des valeurs comparables en valeur absolue à ce qu'on peut observer chez le chamelon en croissance, mais pour un animal qui à l'âge adulte présente des caractéristiques pondérales plus élevées (300 à $400 \mathrm{~kg}$ en moyenne pour une chamelle adulte contre 100 à $250 \mathrm{~kg}$ pour une truie, selon les races). En revanche, compte tenu de la prolificité de la truie (18 à 26 porcelets sevrés / an/truie), sa productivité est sans commune mesure avec celle du dromadaire (un chamelon tous les 2 ans) : $800 \mathrm{~kg} / \mathrm{an} / 100 \mathrm{~kg}$ de poids vif (PV) pour une truie contre 10 à $15 \mathrm{~kg}$ pour un dromadaire.

La productivité laitière, en revanche, serait comparable. En moyenne, elle est de $250 \mathrm{~kg} / \mathrm{an} / 100 \mathrm{~kg}$ PV pour une chamelle et de $225 \mathrm{~kg} / \mathrm{an} / 100 \mathrm{~kg}$ PV pour une truie hyperprolifique de $250 \mathrm{~kg}$. Chez la chamelle, le potentiel laitier pour la consommation humaine est loin d'être négligeable (Faye, 2004), bien que le volume global disponible à l'échelle mondiale (estimé à 5,4 millions de tonnes) ne représente que $0,4 \%$ du volume global de lait consommé par l'homme. Chez la truie, le lait ne sert qu'à alimenter les jeunes porcelets jusqu'au sevrage. Pourtant, 
une truie ayant une portée de 8 à 15 porcelets et devant assurer leur croissance normale jusqu'au sevrage en produit en moyenne 10 à 12 kg/jour.

\section{Des contraintes sanitaires différentes}

Comme pour tous les animaux de rente, les troubles sanitaires représentent une contrainte majeure au développement de l'élevage. De ce point de vue, dromadaires et porcs ont chacun des points faibles, mais ils diffèrent notablement. Les dominantes pathologiques du dromadaire concernent la peau, affectée par des pathologies parasitaires (gale, teigne) ou infectieuses (variole, maladie des abcès), mais le problème dominant est sans hésitation la trypanosomose (à Trypanosoma evansi). Le point faible du porc est l'appareil respiratoire : les épizooties comme les pestes porcines (classique et africaine), la maladie d'Aujeszky, le syndrome dysgénésique respiratoire porcin ou la grippe affectent fortement le secteur porcin. Si le porc est sensible à la fièvre aphteuse, le dromadaire vient d'être retiré de la liste des espèces sensibles par l'Office international des épizooties. Il est cependant un point commun entre les deux espèces, à savoir le parasitisme gastro-intestinal, même si les parasites incriminés divergent également: Strongylus longistipes chez le dromadaire; Metastrongylus, Oesophagostomum et Ascaris suum chez le porc (Permin et al., 1999).

Parmi les zoonoses majeures susceptibles d'être transmises à l'homme, on trouve chez le dromadaire la brucellose, la fièvre de la vallée du Rift (principale contrainte à l'exportation vers les pays du Golfe); chez le porc, la neurocysticercose à Taenia solium (viande ladre), la trichinellose, l'encéphalite japonaise (une arbovirose transmise par un moustique du genre Culex), le virus NIPAH, maladie émergente apparue en Malaisie et entraînant des symptômes neurologiques et respiratoires mortels chez l'homme (Field et al., 2001). Enfin, la transmission de la grippe porcine à l'espèce humaine demeure une question d'actualité.

\section{Des tendances similaires : diversification et lutte contre la pauvreté}

La multifonctionnalité de l'élevage ne se traduit pas de la même façon pour les deux espèces (Tab.). Si le dromadaire relève bien de l'animal multi-usage (Hjort af Ornäs, 1993), fournissant lait, viande, laine, cuir et fumier, utilisé également pour le travail, le loisir ou le sport, le porc, lui, offre une panoplie d'usages beaucoup moins large, mais compensée par la diversité des produits issus de sa chair et de ses abats, la richesse de son lisier et son rôle détritivore.

Le porc est moins un animal de prestige que le dromadaire dans les sociétés d'éleveurs, mais tous deux jouent un rôle essentiel dans les réseaux d'échange. Ainsi, les réseaux Hintila chez les chameliers afar d'Éthiopie $\mathrm{s}^{\prime}$ appuient sur le confiage ${ }^{1}$ de femelles pleines à la parentèle selon des contrats tacites renforçant la solidarité du groupe, tandis que, dans les îles du Pacifique, des réseaux d'entraide familiaux s'appuient sur les échanges coutumiers de porcs selon la logique du don. À Wallis, par exemple, le porc a un poids social et symbolique fort qui apparaît lors des cérémonies traditionnelles, où il est à la fois monnaie d'échange et de rétribution, signe de richesse et fonction d'épargne. À Haïti commeà Madagascar ou au Vietnam, le porc est un moyen d'épargne essentiel, qui constitue une étape primordiale dans les investissements ultérieurs ou dans la sécurisation des ménages.

$\mathrm{Au}$ contraire du porc, le dromadaire semble a priori exclu des processus d'intégration agriculture-élevage. Pourtant, présent depuis longtemps dans les systèmes oasiens pour l'exhaure de l'eau dans les activités maraîchères et les palmeraies, il est de plus en plus utilisé comme auxiliaire de l'agriculture dans les franges des zones désertiques. Aussi le voit-on tirant l'araire (Éthiopie), participant aux travaux de préparation de la terre dans les champs de mil (Niger) ou de maïs (Inde), voire apparié à l'âne dans les systèmes agricoles de l'Atlas (Maroc), ou encore attelé aux norias pour l'extraction de l'huile de sésame (Soudan) ou de tournesol (Inde). Dans ces systèmes intégrant l'énergie cameline à l'activité agricole, l'alimentation, exclusivement à base de pâturages naturels dans les systèmes extensifs, s'enrichit de sousproduits agro-industriels. Certes, de ce point de vue, le porc s'avère un meilleur transformateur des sousproduits de culture (son de riz), des déchets des ménages (eaux grasses, déchets de cuisine, excréments) ou d'agro-industries (résidus d'alcool de riz, pain de soja), mais sa morphologie et son comportement le rendent impropre au travail agricole (à l'exception notable de la recherche truffière). En zone rurale pauvre, il est parfois associé à la pisciculture afin de maximiser les échanges trophiques entre les ateliers de production (Porphyre et Nguyen, 2006 ; Mikolasek et al., 2009).

L'activité d'élevage préserve généralement les populations rurales et périurbaines de la paupérisation par un ensemble de mécanismes (sécurisation, capitalisation, diversification, intégration économique et sociale) pouvant concerner n'importe quelle espèce (Faye, 2001). Dans deux exemples contrastés, dromadaire au Niger et porc au Vietnam, on peut montrer la permanence de ces rôles (Tab.). L'intégration marchande demeure la garantie d'une assignation économique des activités d'élevage, mais la place du formel et de l'informel dans cette économie est variable. Chez les deux espèces, le rôle

\footnotetext{
1 Le terme de « confiage » est employé par les zootechniciens tropicalistes pour évoquer l'action de confier des animaux de son propre troupeau à des parents ou amis (Faye, 2009).
} 
Tableau. Rôle des élevages de dromadaires et de porcs dans la lutte contre la pauvreté, $\mathrm{d}$ 'après les exemples nigérien (dromadaires) et vietnamien (porcs).

\begin{tabular}{|l|l|l|}
\hline & Dromadaires (Niger) & Porcs (Vietnam) \\
\hline Sécurisation & $\begin{array}{l}\text { Sécurisation alimentaire : lait et viande auto- } \\
\text { consommés. } \\
\text { Sécurisation pastorale : valorisation des par- } \\
\text { cours éloignés des points d'eau. }\end{array}$ & $\begin{array}{l}\text { Sécurisation alimentaire : viande. } \\
\text { Sécurisation foncière : valorisation des parcelles agri- } \\
\text { coles faiblement productives (riz), claustration des ani- } \\
\text { maux et lutte contre les vols. }\end{array}$ \\
\hline Capitalisation & $\begin{array}{l}\text { Capitalisation finale : lors de reconstitution } \\
\text { du cheptel après sècheresse, vente de petits } \\
\text { ruminants pour se procurer des droma- } \\
\text { daires. }\end{array}$ & $\begin{array}{l}\text { Épargne sur pied, utilisée pour couvrir les dépenses du } \\
\text { ménage (scolarité, impôts) et rôle d'assurance (rembour- } \\
\text { sement des dettes, rachat de semences); microcrédits } \\
\text { facilement remboursables à court terme. }\end{array}$ \\
\hline Diversification & $\begin{array}{l}\text { Troupeaux multi-espèces. } \\
\text { Diversification des activités (usage agricole). }\end{array}$ & $\begin{array}{l}\text { Associations de pisciculture et d'élevages porcins, épan- } \\
\text { dage des lisiers sur les surfaces agricoles et maraîchères. }\end{array}$ \\
\hline écontégration & $\begin{array}{l}\text { Valorisation marchande du lait de chamelle. } \\
\text { Exportation du cheptel sur pied pour le com- } \\
\text { merce de la viande. }\end{array}$ & $\begin{array}{l}\text { Valorisation commerciale vers les marchés locaux- } \\
\text { régionaux et segmentation des lieux de vente en fonction } \\
\text { des produits (viandes grasses/maigres vers marchés } \\
\text { ruraux/urbains). }\end{array}$ \\
\hline Intégration sociale & $\begin{array}{l}\text { Réseau de solidarité par le confiage* d'ani- } \\
\text { maux. Pouvoir de négociation par l'augmen- } \\
\text { tation de la taille du cheptel. }\end{array}$ & $\begin{array}{l}\text { Dons et échanges de porcelets, structuration des groupe- } \\
\text { ments de petits producteurs. }\end{array}$ \\
\hline
\end{tabular}

*Cf. supra, note 1 .

de l'autoconsommation est considérable, car leur place dans les économies de subsistance demeure centrale, aussi bien dans les régions arides éloignées des circuits marchands (dromadaire) que dans les zones agricoles de montagne, marginalisées par rapport aux circuits d'approvisionnement des villes (montagnes du Vietnam, altiplano péruvien, plateaux malgaches). Chez le dromadaire, le surplus de lait faisait traditionnellement l'objet de dons aux hôtes et aux nécessiteux, mais l'émergence de minilaiteries a suscité la structuration de filières, formelles (Mauritanie, Niger, Asie centrale) ou non (Djibouti, Tchad). Les circuits d'exportation de dromadaires sur pied, particulièrement puissants dans la Corne de l'Afrique, ont également contribué à la structuration de filières d'embouche cameline formelles (Éthiopie et Somalie vers pays du Golfe), avec contractualisation internationalement reconnue (Faye, 2003a), mais s'appuyant aussi sur des circuits informels (Niger et Tchad vers la Lybie, ou Soudan vers l'Égypte).

La production industrielle de porc, en fort développement dans les pays émergents du Sud, passe par l'organisation de schémas génétiques, la professionnalisation des éleveurs, la structuration de la filière, avec, en amont, les fabricants d'aliments pour bétail et, en aval, le secteur de l'abattage et de la transformation des produits. Il est peu d'exemples d'intégration de ce type chez les grands camélidés.

Concernant la question du genre, chez les camélidés, la garde et la gestion des troupeaux est généralement sous l'emprise de l'homme ; mais, dans beaucoup de sociétés, les activités laitières (traite, transformation des produits laitiers, commercialisation) sont gérées majoritairement par les femmes, bien qu'avec l'intégration marchande de ces activités, une tendance à l'emprise masculine s'observe. En Asie centrale, si l'abattage des animaux relève des activités masculines, la préparation des abats est une activité essentiellement féminine. Dans l'élevage traditionnel du porc (Cambodge, Bénin), la femme assure généralement l'entretien et l'alimentation des animaux, et ce sont également de petites détaillantes qui assurent la commercialisation des produits sur les marchés et leur transformation dans la gastronomie locale. Avec l'industrialisation de la production porcine, la femme a su devenir chef d'entreprise à part égale avec les hommes.

\section{Un enjeu commun : la durabilité des élevages}

\section{La tendance à l'intensification}

Les dromadaires, pourtant adaptés aux conditions extensives des zones arides, sont de plus en plus impliqués dans des systèmes de production intensifs. En voici trois exemples: (i) embouche cameline intensive, traditionnelle (Somalie, Éthiopie) pour l'exportation sur pied, modernisée en Tunisie avec la diminution de l'intervalle entre les mises bas, l'allaitement artificiel des chamelons, le sevrage précoce et une alimentation à base de concentrés (Korchani et al., 1997 ; Faye et al., 2002) ; 
(ii) intensification laitière (pays du Golfe, Asie centrale) avec insémination artificielle, transfert d'embryons, sélection des meilleures laitières, traite mécanique, complément alimentaire (Juhasz et Nagy, 2008); (iii) sélection des performances sportives pour la course, avec transfert d'embryons, clonage, alimentation de haute valeur nutritive. Ainsi, en dépit de son adaptation à un écosystème particulier qui le confine aux zones désertiques, le dromadaire montre une plasticité inattendue. Il est de fait capable de s'adapter à une dynamique productiviste visant à satisfaire les besoins en protéines animales des populations urbanisées des régions arides, sur la base de systèmes de production intensifs. Ces changements dans les finalités de la production vers l'intensification des systèmes conduisent à une évolution remarquable du mode d'élevage avec une tendance à la sédentarisation, voire à l'émergence de systèmes hors-sol.

Pour le porc, l'intensification se présente plutôt comme un continuum allant de l'élevage divagant à la production industrielle hors-sol. Ces transitions peuvent être rapides lorsque la demande des marchés augmente localement et que l'aide publique et le secteur privé décident d'investir. Dès lors, la tendance va vers la concentration des animaux, l'augmentation de la taille des cheptels et le regroupement des exploitations dans une même région. La transition inverse est aussi possible: une restructuration de la filière et le retour à un élevage plus économe est observable en cas de surproduction en viande ou lorsque les ressources deviennent limitées, en cas d'épizootie grave ou de crise économique.

\section{Amélioration de la qualité des produits, un enjeu « transversal »}

L'intégration marchande visant l'approvisionnement urbain a suscité partout une augmentation de la demande autant en termes de quantité que de qualité et ce, aussi bien pour les filières porcines que camelines. Toutefois, si les produits laitiers de la chamelle bénéficient a priori d'une notoriété positive liée aux vertus thérapeutiques ou médicinales qu'on leur prête (Konuspayeva et al.,2004), et si la viande de dromadaire est réputée pour sa pauvreté en cholestérol, les produits carnés issus du porc sont marqués du sceau des critiques diététiques et le lard demeure un facteur de risque des affections cardio-vasculaires chez les consommateurs réguliers. À cause des conditions $d$ 'hygiène en élevage, ces produits souffrent également d'une mauvaise réputation quant aux risques bactériologiques et parasitaires. Les qualités nutritionnelles de la viande de porc sont toutefois traditionnellement appréciées grâce à son taux élevé de protéines de valeur biologique reconnue et à ses apports riches et variés en minéraux (fer, phosphore et zinc) et vitamines (B1, B6, B12 et PP).
Bien évidemment, au-delà des contraintes sanitaires forcément spécifiques, des enjeux communs en matière de sécurité des aliments sont à souligner. Les agents pathogènes classiques des intoxications alimentaires, tels que les salmonella, se retrouvent aussi bien dans les produits issus du porc (Le Bas et al., 2006) que dans ceux issus des camélidés (Elmossalami et Yassein, 1994).

\section{Pollution génétique et conservation des races locales}

Une race animale est le produit d'une activité humaine pilotée en fonction d'objectifs de production. De ce point de vue, la pression des éleveurs sur une espèce à vocation extensive comme le dromadaire est demeurée faible. Les noms attribués aux races dans telle ou telle région sont d'ailleurs susceptibles de varier selon les pays ou les ethnies se partageant la zone. C'est dire la grande confusion qui règne dans la nomenclature et l'identification des races de dromadaire. En dépit des progrès de la biologie moléculaire pour spécifier les filiations et identifier les indicateurs génétiques pertinents, la génétique cameline est balbutiante (Schultz et al., 2010).

Les races camelines sont donc vraisemblablement plus proches de populations «naturelles » que de produits issus de sélections raisonnées. L'intervention de l'homme est demeurée superficielle, ce dernier se contentant d'orienter, pour ses besoins de transport, des morphologies adaptées au bât ou à la selle. L'utilisation du terme de race cameline ne manque donc pas d'ambiguïté. Tout au plus peut-on évoquer les tentatives actuelles de sélection de races à vocation laitière, comme l'Al-Majahim en Arabie Saoudite, capable de produire jusqu'à 25-30 litres de lait par jour, ou bien l'Arvana au Turkménistan pour ses performances de croissance (Cherzekov et Saparov, 2005). En conséquence, parler de conservation des races locales camelines consiste d'abord à en faire un réel inventaire sur la base d'outils modernes, comme cela a été tenté en Afrique de l'Est et au Moyen-Orient (Jianlin et al., 2000). Le problème de l'érosion génétique pourrait se poser dans le cas de l'hybridation C. dromedarius x C. Bactrianus en Asie centrale, pratique d'amélioration de la production laitière. Mais, dans la mesure où les hybrides sont trop difficiles à élever à cause de leur caractère, le risque d'absorption est quasi nul (Faye et Konuspayeva, à paraître).

Tout autre est la situation du porc, soumis depuis longtemps à une sélection sur ses performances de croissance et plus récemment sur la qualité du gras. Il existe un risque éminent d'érosion génétique des populations des pays du Sud face aux races améliorées du Nord (Large White, Landrace, Duroc, Piétrain). C'est le cas des populations Mong Cai au Nord Vietnam, menacées d'être remplacées progressivement par les races exotiques (Molenat et Tran, 1991 ; Lemke et al., 2004). 
Du fait de leur meilleure productivité, les races importées supplantent logiquement les races traditionnelles, en dépit de leur rusticité. Néanmoins, par crainte de l'émergence d'une maladie ou de la fragilité des animaux exotiques, nourris avec des rations pauvres, la stratégie des petits producteurs est de croiser ces types d'animaux, bénéficiant ainsi des effets positifs des deux parents. Au Vietnam, sous l'effet d'une diffusion massive de géniteurs améliorateurs exogènes, appuyée par des entreprises multinationales et des organismes d'Etat, des populations porcines moyennement productives des deltas (fleuve Rouge) viennent supplanter les races porcines rustiques (Meo, Muong Khuong) en zones montagneuses. Les noyaux de sélection des races porcines nationales sont ainsi fortement menacés.

\section{Impacts environnementaux}

Les camélidés, en tant qu'herbivores ruminants, participent aux émissions de gaz à effet de serre et ce, dans des proportions importantes à l'échelle individuelle du fait de leur ration à base de fourrages grossiers. Toutefois, leur faible effectif à l'échelle mondiale (moins de $1 \%$ de la biomasse herbivore domestique) leur confère une place très marginale. Par ailleurs, leur dispersion sur des espaces hyperextensifs, leur capacité à valoriser des parcours loin des points d'eau, à utiliser une plus large gamme de ressources pastorales que les autres espèces (Rutagwenda et al., 1989), font qu'ils préservent mieux l'environnement aride que les autres ruminants. En revanche, la concentration des unités de production laitière autour des villes subsahariennes (Nouakchott, Djibouti, Agadez) entraîne des risques de surpâturage et nécessite l'apport de compléments alimentaires (concentrés), ce qui diminue les émissions de gaz à effet de serre, mais crée une compétition avec l'homme. Autrement dit, l'intensification des productions camelines peut avoir des effets environnementaux comparables à ceux des élevages porcins, mais à un degré moindre. Du reste, le fumier de dromadaire est pauvre en azote, contrairement au lisier de porc, qui fournit, selon les valeurs standard, 17,5 kg/an d'azote pour une truie. Dans les élevages porcins concentrés, les lisiers entraînent, en l'absence de moyens de traitement ou de recyclage sur les cultures, une pollution des ressources en eau aussi bien en Asie (Gerber et al., 2005) qu'en Europe (Van der Werf et Petit, 2002). Les impacts majeurs de cette mauvaise gestion des déchets d'élevage porcin sont l'eutrophisation des eaux de surface (détérioration de la potabilité, prolifération d'algues, diminution des populations de poissons), le lessivage des nitrates, voire des organismes pathogènes, dans les eaux souterraines, ainsi que l'accumulation des nutriments, des résidus médicamenteux et des métaux lourds dans les sols. Une utilisation raisonnée des lisiers sur les surfaces agricoles, en substitution des engrais minéraux fabriqués, l'élevage des animaux sur paille ou en plein air sont présentés comme des alternatives. Ce retour à plus d'intégration dans le système agricole semble pouvoir optimiser les revenus des éleveurs tout en réduisant l'atteinte aux biens publics (Porphyre et Nguyen, 2006).

\section{Discussion et conclusion : oppositions ou convergences?}

À bien des égards, comme ces comparaisons l'attestent, les élevages porcin et camelin ne sont guère identiques et leur cohabitation s'avère rarissime. Occupant des écosystèmes opposés, relevant de cultures (religieuses, sociales) plus ou moins incompatibles, dromadaires et cochons sont voués à ne se croiser qu'à la marge. On peut considérer que l'élevage a des fonctions fort différentes chez ces deux espèces : celui du porc, animal à forte productivité (pondérale et numérique), est plus voué à la satisfaction en protéines animales des populations de plus en plus urbanisées de façon rapide et peu coûteuse ; celui du dromadaire est plus tourné vers la valorisation et l'occupation d'espaces non agricoles, apportant aux populations rurales les moyens de leur survie dans ces zones plutôt hostiles grâce à ses capacités d'adaptation et de production. On rattacherait donc volontiers le porc au citadin, dont il partage souvent certains aspects du mode de vie (logement collectif, alimentation du commerce, sédentarité) ; tandis que le dromadaire, animal des grands espaces et des parcours, serait irrémédiablement associé au monde rural dans son mode le plus extensif (mobilité, alimentation naturelle, nomadisme).

Pourtant, les tendances actuelles se traduisent par des trajectoires rapprochant ces deux espèces au travers de modes d'élevage de plus en plus comparables. L'intensification de l'élevage camelin, en particulier dans les pays connaissant un fort développement économique (pays pétroliers), mais aussi autour des cités des régions arides, où les populations récemment urbanisées sont à la recherche de produits rappelant leur culture nomade, est un fait notable. Le triptyque stabulation-concentrés du commerce-sédentarité n'est plus l'apanage des monogastriques comme le porc. À l'inverse, les conséquences néfastes de l'hyperintensification (concentration des lisiers et des déchets), souvent reprochées à l'élevage porcin, ont poussé les producteurs vers des solutions alternatives marquées par une désintensification et un changement dans les pratiques : élevage en plein air, alimentation moins en concurrence avec celle de l'homme, gestion raisonnée des déchets.

Ces évolutions ne valent pas que pour les pays industrialisés, comme en témoignent les réorientations dans les pays du Sud-Est asiatique, par exemple. Elles n'accentuent en aucune manière un rapprochement des deux espèces dans l'espace, car les pratiques socioculturelles 
auxquelles elles sont rattachées sont divergentes. Cependant, elles témoignent de la plasticité des systèmes d'élevage face aux exigences environnementales pour l'un, et d'approvisionnement des villes en milieu aride pour l'autre.

\section{Références}

Alary, V., Faye, B., 2009. Overview of the camel chains in East of Africa: Importance of gaps between the data and the apparent reality, Proceeding of the 2 nd conference of ISOCARD, Djerba (Tunisia), 12-14 March, abstract n 92, 74.

Bengoumi, M., Faye, B., 2002. Adaptation du dromadaire à la déshydratation, Sécheresse, 13, 121-129.

Cherzekov, A., Saparov, G., 2005. The milk productivity of the camel Arvana breed and its use, in Faye, B., Esenov, P. (Eds), Desertification Combat and Food Safety: The Added Value of Camel Producers, Amsterdam, IOS Press 215-220.

Coghlan, A., Mackenzie, D., 2002. Fear over camelpox as bioweapon, New Scientist, 17 April

(http:/ / www.newscientist.com/article/dn2173-fear-overcamelpox-as-bioweapon.html).

Digard, J.-P., 2009. L'Homme et les animaux domestiques: anthropologie d'une passion, Paris, Fayard.

Elmossalami, E., Yassein, N.A., 1994. Salmonellae in slaughtered camels, Archiv für Lebensmittelhygiene, 45, 118120.

Faye, B., 2001. Le rôle de l'élevage dans la lutte contre la pauvreté, Revue d'élevage et de médecine vétérinaire des pays tropicaux, 54, 231-238.

Faye, B., 2003a. Surveillance and control procedures for camel diseases, Regional workshop on Surveillance and control of camels and wildife diseases in the Middle East, Sanaa (Yemen), 10-12 March.

Faye, B., 2003b. La pauvreté, l'élevage et les américains, Les Nouvelles d'Addis, 34, 4.

Faye, B., 2004. Performances et productivité laitière de la chamelle : les données de la littérature, in Lait de chamelle pour l'Afrique : atelier de la filière laitière caméline en Afrique, Niamey, 5-8 novembre 2003, [organisé par la FAO en collaboration avec le Cirad-EMVT et l'ONG nigérienne Karkara], Rome, FAO, 7-14 (http:/ / www.fao.org/docrep/ 010/aj038f/aj038f00.htm).

Faye, B., 2009. Pauvreté et solidarité chez les peuples pastoraux, in Faye, B., Duteurtre, G. (Eds), L'Élevage, richesse des pauvres: stratégies d'éleveurs et organisations sociales face aux risques dans les pays du Sud, Versailles, Quæ, 77-87.

Faye, B., Bengoumi, M., 2000. Le dromadaire face à la sousnutrition minérale : un aspect méconnu de son adaptabilité aux conditions désertiques, Sécheresse, 11, 3, 155-161.

Faye, B., Grech, S., Korchani, T., 2002. Le dromadaire, entre féralisation et intensification, Anthropozoologica, 39, 2, 7-13.

Faye, B., Konuspayeva, G., à paraître. The Encounter between Bactrian Camel and Dromedary Camel in Central Asia: Proceeding of the International Workshop on Camels in Asia and North Africa. Interdisciplinary Perspectives on their
Significance in Past and Present, Vienna (Austria), 5-6 October 2010, Vienna, E. Knoll \& P. Burger.

Field, H., Young, P., Yob, J.M., Mills, J., Hall, L., Mackenzie, J., 2001. The natural history of Hendra and Nipah viruses, Microbes and Infection, 3, 307-314.

Gerber, P., Chilonda, P., Franceschini, G., Menzi, H., 2005. Geographical determinants and environmental implications of livestock production intensification in Asia, Bioresource Technology, 96, 263-276.

Hjort af Ornäs, A. (Ed.), 1993. The Multi-purpose Camel: Interdisciplinary Studies on Pastoral Production in Somalia, Uppsala, EPOS.

Huntington, S., 1997. Le Choc des civilisations, Paris, Odile Jacob. Jianlin, H., Mburu, D., Ochieng, J., Kaufmann, B., Rege, J., Hanotte, O., 2000. Application of New World Camelidae microsatellite primers for amplification of polymorphic loci in Old World Camelids, Animal Genetics, 31, 6, 404-406.

Juhasz, J., Nagy, P., 2008. Challenges in the development of a large-scale milking system for dromedary camels, in Nagy, P., Huszenicza, G., Juhasz, J. (Eds), WBC/ICAR 2008 Satellite Meeting on Camelid Reproduction, 12-13 July, 2008, Budapest, Hungary: Program and Extended Abstracts, 84-87.

Khorchani, T., Ismall, M., Hammadi, M., Moslah, M., Chammem, M., 1997. Sauvegarde du dromadaire et amélioration de sa productivité : bilan des principales recherches menées à l'Institut des régions arides de Médenine (Tunisie), in Actes du séminaire international Acquis scientifiques et perspectives pour un développement durable des zones arides, Médenine, IRA, 368-376.

Konuspayeva, G., Loiseau, G., Faye, B., 2004. La plus-value " santé » du lait de chamelle cru et fermenté : l'expérience du Kazakhstan, Rencontres Recherches Ruminants, 11, 47-50.

Koussou, M.O., Duteurtre, G., 2003. Les facteurs de compétitivité de la filière porcine dans le bassin du Logone, in GIS SYAL (Ed.), Actes du colloque Les Systèmes agroalimentaires localisés : produits, entreprises et dynamiques locales, 16-18 octobre, Montpellier, CD-ROM, Montpellier, Cirad-TERA.

Le Bas, C., Hanh, T.T., Thanh, N.T., Thuong, D.D., Thuy, N.C., 2006. Prevalence and epidemiology of salmonella spp. in small pig abattoirs of Hanoi, Vietnam, Annals of the New York Academy of Sciences, 1081, 269-272.

Lemke, U., Markemann, A., Binh, N.T., Thuy, L.T., Delgado Santivañez, J., Kaufmann, B., Valle Zárate, A., 2004. Set up on-farm performance testing schemes as a component of village breeding programs for pigs in North Vietnam, Deutscher Tropentag 2004, Berlin, October 5-7.

Leterme, P., Buldgen, A., Murgueitio, E., Cuartas, C., 2007. Fodder Banks for Sustainable Pig Production Systems, Cali (Colombia), CIPAV Foundation.

Longo-Hammouda, F., Siboukheur, O.E., Chehma, A., 2007. Aspects nutritionnels des pâturages les plus appréciés par Camelus dromedarius en Algérie, Cahiers Agricultures, 16, 6, 477-483.

Mikolasek, O., Trinh, D.K., Medoc, J.-M., Porphyre, V., 2009. L'intensification écologique d'un modèle de pisciculture intégrée : recycler les effluents d'élevages porcins de la province de Thai Binh (Nord Vietnam), Cahiers Agricultures, 18, 2, 235-241. 
Molenat, M., Tran, T.T., 1991. Génétique et élevage du porc au Viêt-nam, Maisons-Alfort, Institut d'élevage et de médecine vétérinaire des pays tropicaux.

Mopaté, L.Y., Koussou, M.O., Kaboré-Zoungrana, C.Y., 2006. Consommateurs et consommation de la viande porcine en hors-foyer dans la ville de N'Djaména (Tchad), in Parrot, L., Njoya, A., Temple, L., Assogba-Komlan, F., Kahane, R., Ba Diao, M., Havard, M., Actes de l'atelier international Agricultures et développement urbain en Afrique de l'Ouest et du centre, IRAD/INRAB/ISRA/CIRAD, 31 octobre au 3 novembre 2005, Yaoundé (Cameroun), 135-141.

Orwell, G., 1945. Farm Animal, London, Secker and Warburg.

Permin, A., Yelifari, L., Bloch, P., Steenhard, N., Hansen, N.P., Nansen, P., 1999. Parasites in cross-bred pigs in the Upper East Region of Ghana, Veterinary Parasitology, 87, 1, 63-71.

Porphyre, V., Nguyen, Q.C. (Eds), 2006. Pig Production Development, Animal-Waste Management and Environment Protection: A Case Study in Thai Binh Province, Northern Vietnam, Hanoi, PRISE Publications.

Rutagwenda, T., Lechner-Doll, M., Kaske, M., Engelhardt, W.V., Schultka, W., Schwartz, H.J., 1989. Adaptation strategies of camels on a thornbush savannah pasture: Comparison with other domestic animals, Options méditerranéennes, série Séminaires, 2, 69-73.

Schultz, U., Tupac-Yupanqui, I., Martinez, A., Mendez, S., Delgado, J.V., Gomez, M., Dunner, S., Canon, J., 2010. The Canarian camel: A traditional dromedary population, Diversity, 2, 561-571.

Spiegelman, A., 1991. Maus: un survivant raconte, Paris, Flammarion.

Uerpmann, H.P., Uerpmann, M., 2002. The appearance of the domestic camel in SE-Arabia, Journal of Oman Studies, 12, 235-260.

Van der Werf, H.M.G., Petit, J., 2002. Evaluation of the environmental impact of agriculture at the farm level: A comparison and analysis of 12 indicator-based methods, Agriulture, Ecosystems \& Environnement, 93, 131-145.

Vigne, J.-D., 2011. The origins of animal domestication and husbandry: A major change in the history of humanity and the biosphere, Comptes rendus biologies, 334, 171-181.

Yagil, R., 1985. The Desert Camel: Comparartive Physiological Adaptation. Comparative Animal Nutrition, Basel, Karger.

Reçu le 2 juillet 2010. Accepté le 30 juin 2011. 